\title{
APLICAÇÃO DA CADEIA DE MARKOV PARA PREVISÃO MENSAL DE CHUVA EM SANTA CATARINA DURANTE O EL NIÑO OSCILAÇÃO SUL
}

\author{
Rosandro Boligon Minuzzi ${ }^{1}$
}

\begin{abstract}
Resumo: Este estudo objetivou obter matrizes de probabilidade de transição de chuva mensal em Santa Catarina durante eventos do El Niño Oscilação Sul (ENOS). Foram utilizados dados mensais de chuva de setembro a abril do período de 1958 a 2013 de nove estações localizadas em diferentes regiões climáticas do Estado. Para obter as matrizes de probabilidades, as chuvas mensais foram divididas nos seguintes estados: abaixo (-1), dentro (0) e acima (1) da climatologia. $\mathrm{O}$ enquadramento dos valores de chuva mensal nos referidos estados foi baseado nos quantis $\mathrm{Q}_{0,35}$ e $\mathrm{Q}_{0,65}$. Os principais resultados mostram que durante o El Niño há um padrão mais definido das chuvas, resultando em previsões probabilísticas mais eficientes por meio das matrizes de transição, quando comparadas a anos de La Niña. Assim, as matrizes de probabilidade de transição podem ser usadas como auxílio nas previsões mensais de chuva durante eventos do ENOS.
\end{abstract}

Palavras-chave: Climatologia; Matriz de transição; Modelos probabilísticos

\section{APPLICATION OF MARKOV CHAIN FOR THE FORECAST MONTHLY RAINFALL IN SANTA CATARINA DURING EL NIÑO SOUTHERN OSCILLATION}

\begin{abstract}
This study aimed to obtain matrices of transition probability monthly rainfall in Santa Catarina during El Niño Southern Oscillation (ENSO). Were used monthly rainfall data from September to April for the period 1958 to 2013 from nine stations located in different climatic regions of the State. For matrices of probabilities, monthly rainfalls were divided in the following states: below (-1), inside (0) and above (1) climatology. The framework of the values of monthly rainfall in these states was based on quantile $\mathrm{Q}_{0,35}$ and $\mathrm{Q}_{0,65}$. The main results show that during El Niño there is a more defined pattern of rainfall, resulting in more efficient probabilistic forecasts through transition matrices, compared to La Niña years. Thus, the transition probability matrices can be used as an aid in forecasting monthly rainfall during ENSO events.
\end{abstract}

Keywords: Climatology; Transition array; Probabilistic models

\section{APLICACIÓN DE LA CADENA DE MARKOV PARA LA PREVISIÓN MENSUAL DE LLUVIA EN SANTA CATARINA DURANTE EL NIÑO OSCILACIÓN SUR}

Resumen: El objetivo de este estudio fue obtener matrices de probabilidad de la transición de lluvia mensual en Santa Catarina durante los eventos de El Niño Oscilación Sur (ENOS). Fueron utilizados datos mensuales de la lluvia de septiembre a abril entre el periodo de 1958 a

\footnotetext{
${ }^{1}$ Doutor professor, Universidade Federal de Santa Catarina. E-mail: rbminuzzi@hotmail.com
} 
2013 de nueve estaciones localizadas en diferentes regiones climáticas del estado. Para obtener las matrices de probabilidad, las lluvias mensuales fueron divididas en los siguientes estados: abajo (-1), adentro (0) y arriba (1) de la climatología. El encuadramiento de los valores de lluvia mensual en los referentes estados fue basado en los cuantiles C0.35 y C0.65. Los principales resultados muestran que durante EL NIÑO hay un padrón más definido de las lluvias, mostrando previsiones probabilísticas más eficientes por medio de las matrices de transición, cuanto comparadas a los años de La Niña. Así, las matrices de probabilidad de transición pueden ser usadas como auxilio en las previsiones mensuales de lluvia durante eventos del ENOS.

Palabras clave: Climatología; Matriz de transición; Modelos probabilísticos

\section{INTRODUÇÃO}

A ocorrência do El Niño Oscilação Sul (ENOS) é vista como agente de anomalias climáticas em várias regiões do globo, principalmente, na precipitação. Isto se deve, pela grande extensão da região no Pacífico Equatorial onde ocorrem às anomalias da temperatura da superfície do mar (TSM) que caracterizam o fenômeno climático, somado a grande capacidade da água, em transportar energia.

A variação irregular e a persistência em torno das condições normais da temperatura do Pacífico Equatorial revelam duas fases opostas do ENOS. Um desses extremos é representado pelas condições de El Niño, quando se verifica um aquecimento das águas (também denominada fase quente), e a situação oposta, ou seja, quando se verifica um resfriamento das águas (também denominada fase fria), representando condições de La Niña.

Durante a fase quente do ENOS, estudos como de Minuzzi (2010) atesta essa afirmação, destacando o mês de novembro e maio como os mais chuvosos em ano de El Niño de intensidades moderada e forte. Grimm e Ferraz (1998) em uma análise mais abrangente, destacam que em anos La Niña a região Sul do Brasil apresenta anomalias negativas na primavera do ano de início do evento e positivas no ano seguinte.

A climatologia mensal de chuva em Santa Catarina apresenta uma distribuição aproximadamente uniforme, permitindo que a agricultura seja desenvolvida em qualquer época do ano sem a obrigatoriedade da adoção de sistemas de irrigação. Porém, mesmo neste cenário, a chuva é um elemento climático suscetível a grandes variações na escala espaçotemporal, ocasionando maior risco para as atividades agrícolas, em razão da sua falta ou excesso, quando não era o esperado (climatologia).

Grimm (2009) destaca que tanto no verão quanto nas estações de transição, os Complexos Convectivos de Mesoescala (CCM) são frequentes e respondem por grande parte 
da precipitação total na região sul do Brasil. Acrescenta que em grande parte de Santa Catarina, predomina o regime de monções de verão, com máximo de chuva no trimestre dezembro a fevereiro.

Neste contexto, a previsão climática apresenta-se como uma ferramenta fundamental para o turismo, setor elétrico, abastecimento de água, seguradoras rurais, cooperativas e agropecuaristas.

No decorrer dos anos, os pesquisadores tem desenvolvido considerável interesse em modelagem e simulação de chuvas de diferentes maneiras. Uma destas formas são os modelos Markovianos que vêm sendo utilizados nos processos climatológicos que apresentam modelagem sequencial. Wilks (2006) atesta essa informação, destacando que a Cadeia de Markov tem sido usada para caracterizar a transição entre os meses abaixo, próximo e acima da climatologia, como definido pelo Centro de Previsão Climática dos Estados Unidos (CPC). Outro exemplo de aplicação da cadeia de Markov é apresentado por Soares et al. (1999), em estudo objetivando caracterizar a periodicidade das precipitações anuais para determinar a probabilidade de ter, em um período especificado, um número de anos com uma determinada precipitação anual, como subsídios para planejamento florestal, no município de Inhambupe, no estado da Bahia.

Dantas (1998), utilizando a Cadeia de Markov estudaram a distribuição de frequência de períodos secos de diferentes durações no estado da Paraíba. Recorrendo a mesma metodologia estatística, Jimoh e Webster (1996) determinaram a melhor sequência para ocorrência de chuvas diárias na Nigéria, bem como, a melhor sequência de dias úmidos e secos.

Diante das questões expostas, este estudo teve como objetivo obter matrizes de probabilidade de transição da chuva de setembro a abril para o Estado de Santa Catarina em anos do ENOS, como forma de auxiliar as previsões mensais durante a ocorrência do fenômeno climático.

\section{MATERIAL E MÉTODOS}

Para o desenvolvimento deste trabalho foram utilizados dados pluviométricos mensais do período de 1958 a 2013 de nove estações localizadas no Estado de Santa Catarina (Figura 1) e pertencentes à Rede Hidrometeorológica da Agência Nacional de Águas (ANA), de 
forma a representar cada uma, regiões climaticamente homogêneas do Estado definidas por Braga e Ghellre (1999).

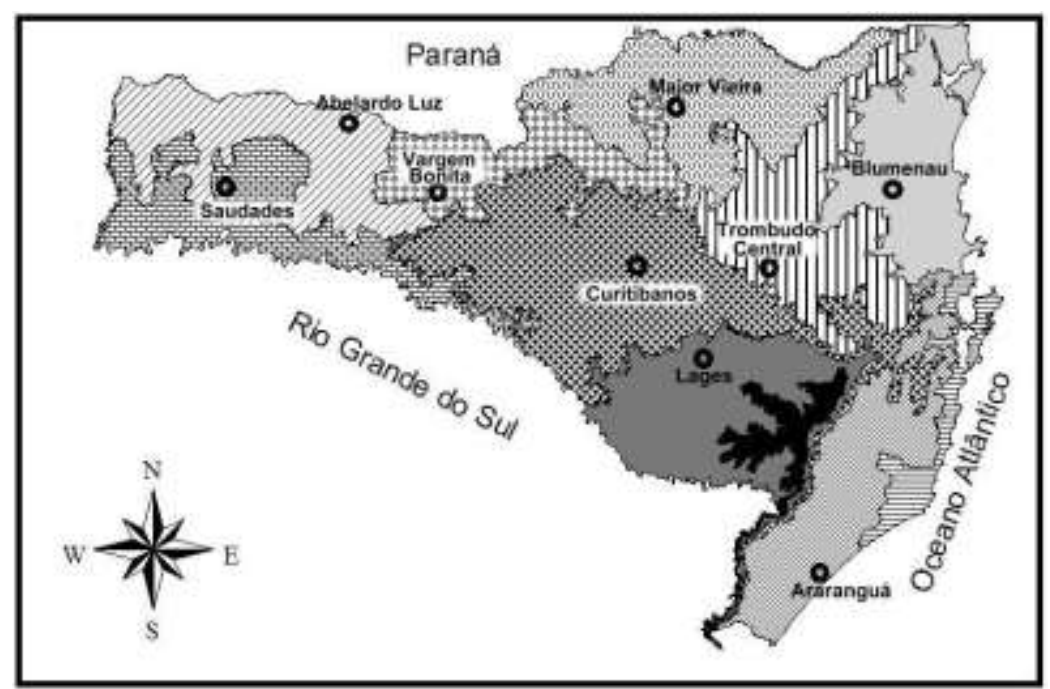

Figura 1. Localização geográfica das estações hidrológicas localizadas nas diferentes regiões climáticas de Santa Catarina e utilizadas no estudo.

O emprego da cronologia das fases do ENOS (Tabela 1) foi definido de acordo com os valores do Índice Niño Oceânico (INO) utilizado pela NOAA (National Oceanic and Atmospheric Administration). O INO foi obtido pela temperatura superficial da região Niño $3.4\left(5^{\circ} \mathrm{N}-5^{\circ} \mathrm{S}\right.$ e $\left.170^{\circ} \mathrm{W}-120^{\circ} \mathrm{W}\right)$, e a NOAA define como ocorrência de El Niño (La Niña), quando o INO fica acima (abaixo) de $0,5{ }^{\circ} \mathrm{C}\left(-0,5{ }^{\circ} \mathrm{C}\right.$ ) por pelo menos cinco trimestres seguidos.

Tabela 1. Cronologia das ocorrências de El Niño e La Niña de 1958 a 2013

\begin{tabular}{llll}
\hline El Niño & $1958 / 59,1963 / 64,1965 / 66,1968 / 69,1969 / 70$, \\
& $1972 / 73,1976 / 77,1977 / 78,1982 / 83,1986 / 87$, \\
& $1987 / 88,1991 / 92,1994 / 95,1997 / 98,2002 / 03$, \\
& $2004 / 05,2006 / 07,2009 / 10$ & & \\
\hline La Niña & $1964 / 65,1970 / 71,1971 / 72,1973 / 74,1974 / 75$, \\
& $1975 / 76,1983 / 84,1984 / 85,1988 / 89,1995 / 96$, \\
& $1998 / 99,1999 / 00,2000 / 01,2005 / 06,2007 / 08$, \\
& $2008 / 09,2010 / 11$ &
\end{tabular}


Para obter a matriz das probabilidades de transição $\left(\mathrm{P}_{\mathrm{ij}}\right)$, na Cadeia de Markov, as chuvas mensais de setembro a abril (período em que geralmente o fenômeno climático está configurado) foram divididas nos seguintes estados: abaixo (-1), dentro (0) e acima (1) da climatologia.

O enquadramento dos valores de chuva (C) mensal nos referidos estados foi baseado nas ordens quantílicas $\mathrm{Q}_{0,35}$ e $\mathrm{Q}_{0,65}$, conforme consta (Xavier, 2001):

Estado -1 (abaixo da média): $\mathrm{C} \leq \mathrm{Q}_{0,35}$

Estado 0 (dentro da média): $\mathrm{Q}_{0,35}<\mathrm{C}<\mathrm{Q}_{0,65}$

Estado 1 (acima da média): $\mathrm{C} \geq \mathrm{Q}_{0,65}$

Assim, os valores que enquadram a chuva nos referidos estados, para cada uma das estações utilizadas no estudo, constam na Tabela 2.

Dessa forma a matriz das probabilidades de transição $\left(\mathrm{P}_{\mathrm{ij}}\right)$ ficou assim definida:

\begin{tabular}{llll}
\hline Estados & -1 & 0 & 1 \\
\hline-1 & $\mathrm{P}_{-1-1}$ & $\mathrm{P}_{-10}$ & $\mathrm{P}_{-11}$ \\
0 & $\mathrm{P}_{0-1}$ & $\mathrm{P}_{00}$ & $\mathrm{P}_{01}$ \\
1 & $\mathrm{P}_{1-1}$ & $\mathrm{P}_{10}$ & $\mathrm{P}_{11}$ \\
\hline $\mathrm{P}_{\mathrm{i}}$ & $\mathrm{P}_{-1}$ & $\mathrm{P}_{0}$ & $\mathrm{P}_{1}$ \\
\hline
\end{tabular}

onde,

$\mathrm{P}_{-1-1}=$ probabilidade da chuva estar no estado -1 , no mês $\mathrm{t}$, e permanecer no estado -1 , no mês $\mathrm{t}+1$

$\mathrm{P}_{-10}=$ probabilidade da chuva estar no estado -1 , no mês $\mathrm{t}$, e passar para o estado 0 , no mês $\mathrm{t}+1$

$\mathrm{P}_{1}=$ probabilidade de estar no estado 1.

As probabilidades de transição $\left(\mathrm{P}_{-1-1}\right.$ a $\left.\mathrm{P}_{11}\right)$ foram calculadas pelas seguintes expressões:

Para $\mathrm{i}=\mathrm{j}$, i e $\mathrm{j}=-1,0$ e 1

$P_{i j}=\frac{n}{N}$

em que, $n$ é o número de meses em que a chuva mensal mudou do estado i, no mês $t$, para o estado j, no mês $\mathrm{t}+1$; e $\mathrm{N}$ é o número de meses de chuva mensal ocorridos no estado $\mathrm{i}$ 
A probabilidade de estar em determinado estado $\left(\mathrm{P}_{\mathrm{i}}\right)$ foi obtida por:

$\mathrm{P}_{\mathrm{i}}=\frac{\mathrm{n}}{\mathrm{N}}$

em que, n é o número de meses de chuva no estado i; e $\mathrm{N}$ é o número total de meses do período (El Niño ou La Niña).

Tabela 2. Valores arredondados mensais de chuva $(\mathrm{mm})$ de setembro a abril obtidos pelos quantis $0,35\left(\mathrm{Q}_{0,35}\right)$ e $0,65\left(\mathrm{Q}_{0,65}\right)$ para os municípios de Vargem Bonita (VB), Abelardo Luz (AL), Saudades (Sau), Curitibanos (Cur), Lages (Lag), Trombudo Central (TC), Araranguá (Ara), Major Vieira (MV) e Blumenau (Blu).

\begin{tabular}{llll}
\hline Jan & Fev & Mar
\end{tabular}

\begin{tabular}{|c|c|c|c|c|c|c|c|c|}
\hline & $\mathrm{Q}_{0,35}$ & $\mathrm{Q}_{0,65}$ & $\mathrm{Q}_{0,35}$ & $\mathrm{Q}_{0,65}$ & $\mathrm{Q}_{0,35}$ & $\mathrm{Q}_{0,65}$ & $\mathrm{Q}_{0,35}$ & $\mathrm{Q}_{0,65}$ \\
\hline VB & 154 & 218 & 140 & 212 & 112 & 145 & 98 & 169 \\
\hline AL & 130 & 206 & 130 & 193 & 104 & 181 & 109 & 163 \\
\hline Sau & 129 & 182 & 116 & 169 & 86 & 157 & 107 & 162 \\
\hline Cur & 128 & 181 & 119 & 180 & 83 & 128 & 65 & 124 \\
\hline Lag & 120 & 192 & 136 & 169 & 92 & 135 & 79 & 124 \\
\hline $\mathrm{TC}$ & 140 & 196 & 129 & 173 & 96 & 130 & 55 & 108 \\
\hline Ara & 102 & 176 & 127 & 176 & 115 & 174 & 71 & 99 \\
\hline MV & 128 & 183 & 127 & 177 & 93 & 145 & 63 & 111 \\
\hline Blu & 172 & 228 & 162 & 236 & 135 & 180 & 75 & 114 \\
\hline
\end{tabular}

\begin{tabular}{lcccccccc} 
& $\mathrm{Q}_{0,35}$ & $\mathrm{Q}_{0,65}$ & $\mathrm{Q}_{0,35}$ & $\mathrm{Q}_{0,65}$ & $\mathrm{Q}_{0,35}$ & $\mathrm{Q}_{0,65}$ & $\mathrm{Q}_{0,35}$ & $\mathrm{Q}_{0,65}$ \\
\hline VB & 136 & 199 & 174 & 219 & 113 & 185 & 136 & 198 \\
AL & 134 & 196 & 159 & 248 & 103 & 172 & 129 & 177 \\
Sau & 86 & 150 & 127 & 194 & 99 & 161 & 111 & 143 \\
Cur & 101 & 165 & 130 & 186 & 92 & 147 & 110 & 168 \\
\hline
\end{tabular}




\begin{tabular}{lcccccccc}
\hline Lag & 121 & 178 & 113 & 170 & 98 & 142 & 98 & 133 \\
TC & 110 & 152 & 117 & 178 & 87 & 129 & 97 & 167 \\
Ara & 91 & 154 & 85 & 131 & 85 & 129 & 79 & 131 \\
MV & 126 & 172 & 138 & 182 & 93 & 133 & 105 & 164 \\
Blu & 111 & 158 & 115 & 153 & 80 & 133 & 123 & 162 \\
\hline
\end{tabular}

\section{RESULTADOS E DISCUSSÃO}

As informações da Tabela 3 mostram as probabilidades da chuva mensal estar abaixo (-1), dentro (0) ou acima (1) da climatologia durante um evento de El Niño, com base no estado de chuva observado no mês corrente. Como exemplo, se em setembro a chuva na região de Vargem Bonita esteve abaixo da climatologia (inferior a $136 \mathrm{~mm}$, conforme Tabela 2), há uma maior probabilidade (37\%) da chuva de outubro ficar dentro do esperado para o mês, ou com valor entre 174 e $219 \mathrm{~mm}$ (Tabela 2). Outra forma de apresentar os resultados, habitualmente utilizados nas previsões climáticas, envolve a probabilidade da chuva ficar dentro da climatologia, juntamente com a probabilidade indicada do estado -1 ou 1 . Como exemplo, se em ano de El Niño a chuva de novembro em Blumenau esteve dentro do esperado (estado 0), pode-se afirmar que em dezembro há $69 \%$ de probabilidade da chuva ficar entre a climatologia (30\%) e acima desta (39\%), ou 69\% de chover mais do que $123 \mathrm{~mm}$ e apenas $16 \%$ que a chuva fique abaixo da climatologia (estado -1).

Tabela 3 - Matriz de probabilidade de transição (\%) para os estados da chuva mensal abaixo (1), dentro (0) e acima (1) da climatologia durante eventos do El Niño nos municípios utilizados no estudo.

\begin{tabular}{lcccccccccc} 
& \multicolumn{3}{c}{ Vargem Bonita } & \multicolumn{3}{c}{ Saudades } & \multicolumn{3}{c}{ Curitibanos } \\
\hline Estado & -1 & 0 & 1 & -1 & 0 & 1 & -1 & 0 & 1 \\
& & & & & & & & & \\
\hline-1 & 23 & 37 & 26 & 27 & 13 & 38 & 23 & 26 & 37 \\
0 & 28 & 23 & 38 & 31 & 28 & 31 & 40 & 21 & 29
\end{tabular}




\begin{tabular}{cccccccccc}
1 & 24 & 17 & 48 & 20 & 30 & 38 & 18 & 29 & 38 \\
\hline $\mathrm{P}_{\mathrm{i}}$ & 31 & 28 & 41 & 29 & 30 & 40 & 31 & 30 & 39 \\
\hline & \multicolumn{3}{c}{ Lages } & \multicolumn{3}{c}{$\begin{array}{c}\text { Trombudo } \\
\text { Central }\end{array}$} \\
& & \multicolumn{3}{c}{ Araranguá }
\end{tabular}

\begin{tabular}{|c|c|c|c|c|c|c|c|c|c|}
\hline Estado & -1 & 0 & 1 & -1 & 0 & 1 & -1 & 0 & 1 \\
\hline-1 & 18 & 32 & 46 & 24 & 24 & 39 & 20 & 23 & 40 \\
\hline 0 & 30 & 22 & 30 & 36 & 16 & 44 & 25 & 25 & 31 \\
\hline 1 & 39 & 22 & 33 & 24 & 28 & 46 & 25 & 36 & 30 \\
\hline$P_{i}$ & 32 & 26 & 41 & 30 & 31 & 39 & 28 & 34 & 38 \\
\hline & \multicolumn{3}{|c|}{ Major Vieira } & \multicolumn{3}{|c|}{ Blumenau } & \multicolumn{3}{|c|}{ Abelardo Luz } \\
\hline Estado & -1 & 0 & 1 & -1 & 0 & 1 & -1 & 0 & 1 \\
\hline-1 & 36 & 21 & 31 & 33 & 23 & 33 & 26 & 35 & 26 \\
\hline 0 & 29 & 29 & 29 & 16 & 30 & 39 & 27 & 33 & 29 \\
\hline 1 & 23 & 35 & 28 & 21 & 30 & 38 & 25 & 17 & 42 \\
\hline $\mathrm{P}_{\mathrm{i}}$ & 35 & 32 & 33 & 29 & 32 & 39 & 31 & 32 & 37 \\
\hline
\end{tabular}

Com exceção de Major Vieira, nos demais municípios analisados há uma maior ocorrência $\left(\mathrm{P}_{\mathrm{i}}\right)$ de meses chuvosos (estado 1, entre $38 \%$ a $41 \%$ ) de setembro a abril durante eventos do El Niño. Provavelmente, por este motivo, há muitas situações em que as maiores probabilidades são de chuva acima da climatologia (estado 1). Quando a chuva observada fica abaixo do esperado (estado -1), a probabilidade da chuva passar para o estado 1 é maior em cinco municípios (Saudades, Trombudo Central, Curitibanos, Araranguá e Lages), chegando a 46\% para Lages. Já, quando a chuva fica dentro da climatologia, em quatro municípios a maior probabilidade é de que a chuva passe para o estado 1. Mas o mais relevante, é quando a chuva observada fica acima da climatologia e a probabilidade de se manter neste estado é maior em Blumenau, Saudades, Curitibanos, Abelardo Luz, Trombudo Central e Vargem Bonita, havendo nestes três últimos municípios, probabilidades de $42 \%, 46 \%$ e $48 \%$, respectivamente. 
Há algumas situações em que a igualdade entre os maiores valores probabilísticos encontrados para um dado estado de chuva observado, dificultam a sua utilização para fins de previsão climática. Como exemplos, em Blumenau quando a chuva fica abaixo da climatologia, a probabilidade de se manter no estado -1 ou passar para o estado 1 é de $33 \%$, enquanto em Major Vieira, Saudades e Lages a igualdade entre os referidos estados ocorre quando a chuva observada fica dentro da climatologia.

O regime de chuva de setembro a abril durante eventos de La Niña são mais divergentes entre as diferentes regiões climáticas de Santa Catarina, conforme observado na Tabela 4. Isto resulta na maior ineficiência da utilização das matrizes de transição para previsão climática de chuva, em especial, nas regiões dos municípios de Saudades, Curitibanos, Vargem Bonita e Araranguá. Neste último município, a ocorrência de meses com chuva abaixo e acima da climatologia é igual (37\%), assim como, as maiores probabilidades serem nos estados 1 e -1 e semelhantes entre si, independente do estado de chuva observado.

$\mathrm{Na}$ contrapartida, há matrizes de transição em que os seus valores viabilizam a sua utilização para previsão climática, mesmo que o percentual de ocorrências $\left(\mathrm{P}_{\mathrm{i}}\right)$ entre alguns estados de chuva sejam semelhantes. Em Abelardo Luz, apesar da igualdade nas ocorrências de chuvas abaixo e acima da climatologia (39\%) em eventos La Niña, quando a chuva no mês corrente fica abaixo, dentro ou acima da climatologia, a probabilidade maior é de que a chuva no mês seguinte, se mantenha no estado -1 (42\%), fique dentro (40\%) e acima do esperado (42\%), respectivamente. Ressalta-se também os resultados para Trombudo Central, onde para qualquer estado que seja observado, a maior probabilidade é de que a chuvas fiquem no estado 1.

Tabela 4 - Matriz de probabilidade de transição (\%) para os estados da chuva mensal abaixo (1), dentro (0) e acima (1) da climatologia durante eventos do La Niña nos municípios utilizados no estudo.

\begin{tabular}{llllllllll} 
& \multicolumn{3}{c}{ Vargem Bonita } & \multicolumn{3}{c}{ Saudades } & \multicolumn{3}{c}{ Curitibanos } \\
\hline Estado & -1 & 0 & 1 & -1 & 0 & 1 & -1 & 0 & 1 \\
\hline-1 & 32 & 32 & 23 & 27 & 24 & 38 & 37 & 23 & 26 \\
0 & 37 & 16 & 35 & 32 & 29 & 22 & 31 & 29 & 23 \\
1 & 24 & 31 & 31 & 33 & 22 & 35 & 20 & 34 & 34
\end{tabular}




\begin{tabular}{|c|c|c|c|c|c|c|c|c|c|}
\hline$P_{i}$ & 33 & 30 & 36 & 35 & 27 & 38 & 34 & 33 & 33 \\
\hline & \multicolumn{3}{|c|}{ Lages } & \multicolumn{3}{|c|}{ Trombudo } & \multicolumn{3}{|c|}{ Araranguá } \\
\hline & \multicolumn{9}{|c|}{ Central } \\
\hline Estado & -1 & 0 & 1 & -1 & 0 & 1 & -1 & 0 & 1 \\
\hline-1 & 29 & 21 & 35 & 24 & 24 & 39 & 33 & 23 & 35 \\
\hline 0 & 41 & 27 & 24 & 36 & 16 & 44 & 33 & 27 & 30 \\
\hline 1 & 25 & 21 & 39 & 24 & 28 & 46 & 29 & 17 & 33 \\
\hline \multirow[t]{2}{*}{$P_{i}$} & 34 & 26 & 40 & 33 & 31 & 35 & 37 & 26 & 37 \\
\hline & \multicolumn{3}{|c|}{ Major Vieira } & \multicolumn{3}{|c|}{ Blumenau } & \multicolumn{3}{|c|}{ Abelardo Luz } \\
\hline Estado & -1 & 0 & 1 & -1 & 0 & 1 & -1 & 0 & 1 \\
\hline-1 & 32 & 24 & 27 & 39 & 20 & 25 & 42 & 18 & 24 \\
\hline 0 & 25 & 28 & 40 & 24 & 43 & 26 & 40 & 13 & 33 \\
\hline 1 & 26 & 23 & 38 & 23 & 23 & 38 & 24 & 22 & 42 \\
\hline$P_{i}$ & 31 & 30 & 40 & 31 & 32 & 37 & 39 & 21 & 39 \\
\hline
\end{tabular}

Nota-se que há diferença no regime de chuva mensal entre as ocorrências de El Niño e La Niña. Durante a fase quente há um padrão mais definido das chuvas, havendo maior frequência de meses com chuva acima da climatologia durante o período de setembro a abril e, predominantemente com maiores probabilidades do mês $\mathrm{t}+1$ em ficar dentro (estado 0 ) ou acima da climatologia (estado 1). Manson e Goddard (2001) chamaram a atenção que as respostas das fases do ENOS em muitas áreas nem sempre são contrárias, e os eventos de La Niña afetam 5\% a 15\% mais áreas continentais do que durante eventos El Niño.

No que tange a tendência chuvosa em Santa Catarina Grimm e Tedeschi (2004) relataram que em episódios El Niño, o número de eventos extremos de precipitação tende a aumentar no Centro-Sudeste do Brasil durante a primavera. Em eventos La Niña as autoras notaram um comportamento oposto na região, especialmente em outubro e novembro do ano de início da forçante climática, ocorrendo diminuição expressiva em janeiro no número de eventos extremos. 


\section{CONCLUSÕES}

As matrizes de probabilidade de transição obtidas para diferentes regiões climáticas de Santa Catarina podem ser usadas como auxílio nas previsões mensais de chuva durante eventos do ENOS.

Durante o El Niño há um padrão mais definido das chuvas, resultando em previsões probabilísticas mais eficientes por meio das matrizes de transição, quando comparadas a anos de La Niña.

\section{REFERÊNCIAS BIBLIOGRÁFICAS}

BRAGA, H.J.; GHELLRE, R. Proposta de diferenciação climática para o Estado de Santa Catarina. In: CONGRESSO BRASILEIRO DE AGROMETEOROLOGIA, 11 E REUNIÃO LATINO-AMERICANA DE AGROMETEOROLOGIA, 2, 1999, Florianópolis. Anais... Florianópolis: SBAgro, 1999. CD-Rom

DANTAS, P.R.C. Frequency distribution of dry speels at some stations en Paraíba. In: CONGRESSO BRASILEIRO DE METEOROLOGIA 11; Brasília. Anais...Brasília, 1998.

GRIMM, A.M. Clima da região Sul do Brasil. In: CAVALCANTI et al. (Org.) Tempo e clima no Brasil. São Paulo, SP: Oficina de Texto, 2009. p.259-275.

GRIMM, A.M.; FERRAZ, S.E.T. Sudeste do Brasil: uma região de transição no impacto de eventos extremos da Oscilação Sul. Parte II: La Niña. In: CONGRESSO BRASILEIRO DE METEOROLOGIA, 10, 1998, Brasília. Anais... Brasília, DF: SBMet, 1998. CD-Rom.

GRIMM, A.M.; TEDESCHI, R.G. Influência de eventos El Niño e La Niña sobre a frequência de eventos extremos de precipitação no Brasil. In: CONGRESSO BRASILEIRO DE METEOROLOGIA, 13, 2004, Fortaleza. Anais... Fortaleza, CE: SBMet, 2004. CD-Rom. JIMOH O. D., WEBSTER, P. The optimum order of a Markov chain model for daily rainfall in Nigéria. Journal of Hydrology, n.185, p.45-69, 1996.

MANSON, S.J.; GODDARD, L. Probabilistic precipitation anomalies associated with ENSO. Bulletim of the American Meteorological Society, Washington, v.82, n.4, p.619-638, 2001. MINUZZI, R.B. Chuvas em Santa Catarina durantes eventos do El Niño Oscilação Sul. Geosul, v.25, n.50, p.107-127. 2010.

SOARES, C.P.B. et al. Caracterização da periodicidade e da probabilidade de ocorrência de precipitações anuais como subsídio para o planejamento florestal. Revista Árvore, v. 22 , n. 3, p. $315-324,1998$.

XAVIER, T. de. M.B.S. Tempo de chuva - Estudos climáticos e de previsão para o Ceará e Nordeste Setentrional. Fortaleza: ABC Editora, 2001. 478p.

WILKS, D.S. Statistical methods in the atmospheric sciences. $2^{\circ}$ ed. San Diego, EUA: Elsevier, 627p. 2006.

Recebido em setembro de 2014.

Aceito em março de 2017. 